\title{
Keto-Enol-Umwandlung des Acetylacetons im Gaszustand ${ }^{1}$
}

\author{
Von Walter Strohmeier und Irmtraud Höhne \\ Aus der Physikalisch-chemischen Abteilung des Chemischen Instituts der Universität Würzburg \\ (Z. Naturforschg. 7 b, 184-187 [1952]; eingegangen am 16. November 1951)
}

Es wurden die thermodynamisch stabilen Keto-Enol-Gleichgewichte des Acetylacetons in der Gasphase in einem großen Temperaturbereich gemessen.

Es wird eine gegenüber der II. Mitt. ${ }^{1}$ verbesserte und vereinfachte Apparatur zur Messung der K-E-Gasgleichgewichte beschrieben.

Aus den gemessenen K-E-Gleichgewichten wurden die thermodynamischen Daten $\Delta G, \Delta H$, $\Delta S$ und $\Delta C_{p}$ der K-E-Umwandlung im Gaszustand berechnet 2. Die für die Enolisierungstendenz maßgebenden thermodynamischen Größen des Acetylacetons werden mit dèm des Acetessigesters verglichen.

$\mathrm{I}^{\mathrm{n}}$ n der II. Mitt. wurde eine Apparatur beschrieben, welche es nach dem Prinzip der isothermen Destillation im Hochvakuum ermöglicht, von tautomeren Systemen die thermodynamisch stabilen Gasgleichgewichte über einer kondensierten Mischphase in einem großen Temperaturbereich zu bestimmen. Diese Apparatur wurde jetzt noch verbessert und vereinfacht, wie im folgenden beschrieben wird. Es wurden K-E-Gleichgewichtsmessungen am Acetylaceton im Gaszustand ausgeführt.

Aus den gemessenen K-E-Gleichgewichten werden die thermodynamischen Daten $\Delta G, \Delta H, \Delta S$ und $\Delta C_{p}$ der Keto-Enol-Umwandlung im Gaszustand in einem größeren $T$-Bereich angegeben.

An der in der II. Mitt. beschriebenen Apparatur zeigte sich, daß bei Serienmessungen die Verwendung von zwei Temperaturbädern zeitraubend und umständlich ist. Außerdem war es notwendig, eine genaue Regulierung der Destillationsgeschwindigkeit zu ermöglichen. Auch die zeitraubende „Endtitrationsmethode“ konnte umgangen werden.

\section{a) A p paratur (Abb. 1) ${ }^{3}$}

Die gegenüber Mitt. II veränderte Apparatur aus Jenaer Glas ist in sich geschlossen. Die Badflüssigkeit (Paraffinöl) wird von einem Höppler-Thermostaten bei $\mathrm{Z}$ in die Apparatur hineingepumpt. Sie umspült die Düse D und heizt beim Durchströmen des Mantelrohrs Ma die gesamte Apparatur. Die Substanz wird durch den Stutzen E eingefüllt und befindet sich in $\mathrm{S}$. Die Haube $\mathrm{H}$ ist elektrisch

1 Die Mitteilungen über die K-E-Umwandlung im Gaszustand und in Lösungen werden folgendermaßen bezeichnet: I. Mitt.: G. Bri e gle b u. H. Rebele in, Z. Naturforschg. 2a, 562 [1947]. II. Mitt.: W. S tro h meier u. G. Briegleb, ebenda 6 b, 1 [1951]. III. Mitt.: G. B ri e g le b u. W. S tro h m e i e r, ebenda $6 \mathbf{b}, 6$ [1951]. heizbar und wird auf die Temperatur der Badflüssigkeit aufgeheizt (Thermometer $\mathrm{T}_{\mathrm{o}}$ ). Die Substanz wird mit dem magnetisch angetriebenen Rührer R kräftig durchgerührt. Am unteren Teil des Stempels St des KPG-Schliffes befindet sich eine Kovar-Metalleinschmelzung, an welcher die vernickelte Stahlfeder $\mathrm{F}$ befestigt ist, die an ihrem unteren Ende, durch eine 2. Kovar-Metalleinschmelzung das Kernstück cles Glasventiles V trägt. Das Ventil V ist vakuumdicht eingeschliffen und wird nicht durch „Drehen“, sondern durch Andrücken geschlossen. An dem oberen Ende des Stempels St ist ein Mikrogewinde M so befestigt, daß die Drehung an $\mathrm{M}$ in eine vertikale Bewegung übergeführt wird. Dadurch wird eine sichere und saubere Feineinstellung des Ventils V gewährleistet. Damit das zur Dichtung benutzte $\mathrm{Hg}$ nicht durch den KPG-Schliff gedrückt wird, muß in $\mathrm{C}$ annähernd derselbe Druck wie in der Apparatur eingestellt werden. Druckgleichheit wird an den beiden Manometern $\mathrm{M}_{1}$ und $\mathrm{M}_{2}$ abgelesen. Der Stempel St ist durch die eingeschliffenen Messinghülsen $L_{1}$ und $L_{2}$, welche mit den Gummischläuchen $G_{1}$ und $G_{2}$ befestigt sind, beweglich und luftdicht angeschlossen. Das Kondensationsgefäß $\mathrm{O}$ ist durch den Schliff $\mathrm{J}_{1}$ mit der Apparatur und den Schliff $\mathrm{J}_{2}$ mit dem Hochvakuum verbunden und kann somit leicht zur Analyse der bei B einkondensierten Substanz abgenommen werden.

\section{b) Verlauf der Messung}

Die Substanz in $\mathrm{S}$ wird mit einer Spur $\mathrm{Ba}(\mathrm{OH})_{2}$ versetzt, damit sich in der flüssigen Phase das K-E-Gleichgewicht immer wieder schnell genug einstellt. Die Appa-

2 Symbole: $\Delta C_{p}$ : Molwärmenänderung bei der K-EUmwandlung; $\vartheta=273^{\circ} \mathrm{K} ; \mathrm{E} \equiv \mathrm{Enol} ; \Delta G=-R T \ln \mathfrak{\Omega}$ : freie Enthalpieänderung bei der K-E-Umwandlung; $\Delta H$ : Enthalpieänderung bei der $\mathrm{K}-\mathrm{E}-\mathrm{U}$ mwandlung; $\mathrm{K} \equiv \mathrm{Ke}-$ ton; $\Re=\xi_{\mathrm{E}} / \xi_{\mathrm{K}}$ : Gleichgewichtskonstante der K-E-Umwandlung; $R=$ Gaskonstante; $\Delta S$ : Enthalpieänderung bei der K-E-Umwandlung. $T$ : absol. Temperatur. $\xi_{\mathrm{E}}$ und $\xi_{\mathrm{K}}$ : Molenbrüche an $\mathrm{E}$ und $\mathrm{K}$.

3 Herrn Glasbläser Amen de danken wir für die sehr viel Geschicklichkeit erfordernde Anfertigung der Glasapparatur. 
ratur ist mit Luft gefüllt und das Ventil V geschlossen. Man pumpt die Badflüssigkeit von der gewünschten Temperatur, z. B. $50^{\circ}$, durch die Apparatur. Die Substanz wird mit dem Magnetrührer kräftig durchgerührt. Das Kondensationsgefäß $\mathrm{O}$ taucht in flüssige Luft. Nachdem sich die Temperatur eingespielt hat, pumpt man von der Hochvakuumseite H.V ab und öffnet dann das Ventil V langsam durch Drehen an der Mikrometerschraube M. Durch regulierendes Bedienen der Hähne $\mathrm{H}_{1}$ bzw. $\mathrm{H}_{2}$ erreicht

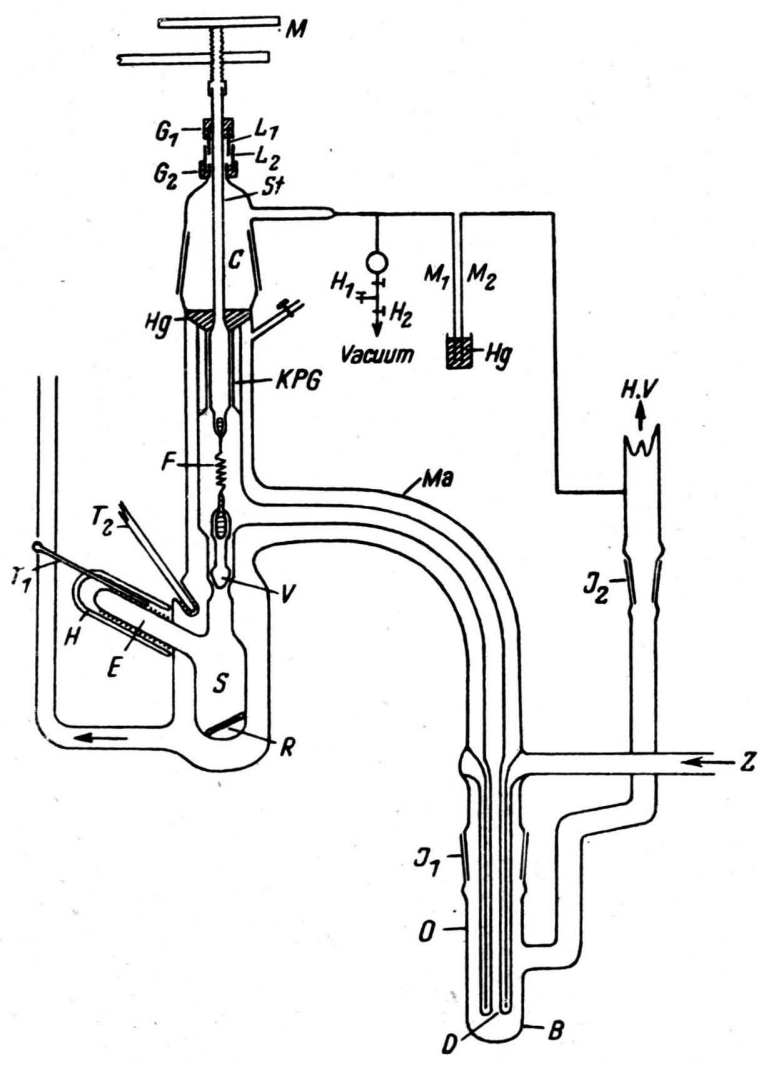

Abb. 1. Apparatur zur Bestimmung des K-E-Gleichgewichtes im Gas, im thermodynamischen Gleichgewicht mit der kondensierten Phase in größeren $T$-Bereichen.

man leicht, daß die Manometer $\mathrm{M}_{1}$ und $\mathrm{M}_{2}$ ungefähr denselben Druck anzeigen. (Damit sich das $\mathrm{Hg}$ in $\mathrm{C}$ nicht durch den Schliff drückt.) Die Substanz strömt mit einer regulierten Geschwindigkeit durch die Düse D und wird auf der mit flüssiger Luft gekühlten Fläche B festgefroren. Die Destillationsgeschwindigkeit läßt sich leicht am Grad des „Kochens“ der flüssigen Luft (durch die freiwerdende Kondensationswärme) abschätzen. Bei niedrigerer Versuchstemperatur und somit kleinem Dampfdruck der Substanz in S wird während der ganzen Destillationszeit abgepumpt; bei hohem Dampfdruck der Substanz nur so lange, bis die Destillation mit der gewünschten Geschwindigkeit einsetzt. c) Bestimmung der Gesamtmenge des überdestillierten festen Esters

Weil die Bestimmung der Gesamtmenge des überdestillierten Esters nach der in Mitt. II beschriebenen „Endtitrationsmethode“ zeitraubend und umständlich ist, wurde nach dem folgenden Prinzip verfahren:

Waren ungefähr $1-2 \mathrm{~g}$ Substanz im Gefäß $\mathrm{O}$ bei $-180^{\circ}$ einkondensiert, so wurde dieses von der Apparatur abgezogen und die Substanz mit einer auf $-180^{\circ}$ gekühlten Spachtel vom Boden B losgelöst. Durch Kippen des Gefäßes O wurde die feste Substanz in das auf $-80^{\circ}$ gekühlte Wägeglas $W_{1}$ gefüllt (Abb. 2). $W_{1}$ stand über $\mathrm{P}_{2} \mathrm{O}_{5}$ auf einem Glasfuß in einem großen Wägeglas $W_{2}$. $\mathrm{W}_{2}$ wurde durch Eintauchen des Drahtgestelles D in ein Kältebad auf $-80^{\circ}$ gekühlt.

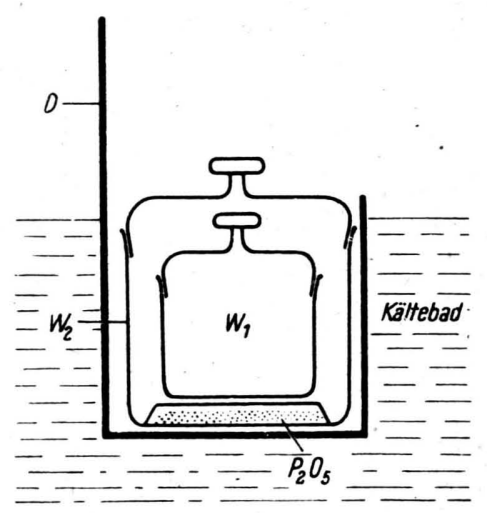

Abb. 2. Anordnung zur Wägung des aus der Gasphase in $\mathrm{O}$ (Abb. 1) kondensierten K-E-Gemisches.

War clie Substanz in $W_{1}$ eingekippt, so wurde das Wägeglas $W_{1}$ unmittelbar anschließend gewogen. Man konnte auf $0,1 \mathrm{mg}$ genau wiegen. Im Waagengehäuse befand sich $\mathrm{P}_{2} \mathrm{O}_{5}$, um ein Beschlagen des Wägeglases mit Wasser zu vermeiden ${ }^{4}$. Da die Wägung mit automatischer Gewichtsauflage nie länger als 1 Min. dauerte, war die Substanz nach dem Wägen noch fest. Nach dem Wägen wurde die noch feste Substanz samt Wägeglas in $30 \mathrm{ccm}$ Methanol von $-20^{\circ}$ geworfen und mit einer eingestellten Lösung von Brom in Methanol titriert. Es war unbedingt erforderlich, daß die Substanz noch fest war, wenn sie in das Titrationsgefäß gegeben wurde, da sich flüssiges Acetylaceton von hohem Enolgehalt (über 90\%) auch bei tiefen Temperaturen sehr schnell umlagert. Mit der Titration wurde begonnen, wenn das eingeworfene Acetylaceton gerade an der Oberfläche zu schmelzen und sich somit im Methanol zu lösen begann.

4 Trotz aller Vorsichtsmaßnahmen ließ es sich nicht vermeiden, daß sich das gekühlte Wägeglas mit einer feinen Wasserhaut beschlug. Durch eine Reihe von Blindversuchen wurde festgestellt, daß diese bei den vorgesehenen Arbeitsbedingungen konstant zwischen 8 und $10 \mathrm{mg}$ betrug. Es wurden daher von der Einwaage $(\approx 1500 \mathrm{mg})$ $9 \mathrm{mg}$ abgezogen, um das Gewicht des festen Acetylacetons $\mathrm{zu}$ bekommen. 
In der Literatur 5 wurde der Einwand erhoben, daß möglicherweise nicht der wahre Enolgehalt titriert wird, da sich ein Teil des Enols durch Anlagerung von hydroxylhaltigen Lösungsmitteln an die Doppelbindung nach Art eines Hydrates bzw. Acetals der Titration entziehen könne.

Um diesen Einwand zu klären, wurde flüssiges Acetylaceton bekannten Enolgehaltes in Methanol, Äther und Hexan von $-20^{\circ}$ gelöst und dann anschließend mit der direkten Bromtitrationsmethode der Enolgehalt bestimmt. In allen drei Fällen war der Enolgehalt der gleiche. Bei dem von uns angewandten Titrationsverfahren ist also der experimentell gemessene Enolgehalt unabhängig von dem Lösungsmittel, in welchem titriert wird.

\section{d) Meßergebnisseder Keto-Enol- Umwandlung des Acetylacetons im $\mathrm{G}$ as zustand}

Die Ergebnisse der Messungen am Acetylaceton im $T$-Bereich von $0^{\circ}$ bis $120^{\circ}$ zeigt Tab. 1. Diese Werte sind Mittelwerte aus jeweils mindestens 3 Einzelbestimmungen.

\begin{tabular}{|c|c|c|c|}
\hline$T$ & $\% / \mathrm{E}$ & $\begin{array}{c}\mathfrak{\Omega}_{\mathrm{K} \rightarrow \mathrm{E}} \\
\text { exp. }\end{array}$ & $\begin{array}{c}\log \mathfrak{K} \\
\text { exp. }\end{array}$ \\
\hline$(1)$ & $(2)$ & $(3)$ & $(4)$ \\
273,2 & 95 & 19,0 & 1,278 \\
325,2 & 91 & 10,12 & 1,005 \\
359,2 & 87,4 & 6,94 & 0,841 \\
388,2 & 83 & $4,88_{5}$ & 0,688 \\
\hline
\end{tabular}

Tab. 1. Meßergebnisse der $\mathrm{K} \rightarrow \mathrm{E}$-Umwandlung von Acetylaceton im Gaszustand.

Die experimentellen Ergebnisse lassen sich innerhalb der Meßgenauigkeit durch die Formel

$$
\begin{aligned}
\log \mathfrak{\Omega}_{\mathrm{K} / \mathrm{E}}= & -\frac{\Delta H_{\vartheta}}{2,303 \cdot R} \cdot \frac{1}{T} \\
& +\frac{\Delta C_{p}}{R}\left(\log T+\frac{\vartheta}{2,303 T}\right)+{\text {. } \text { onst. }^{6}}^{6} \\
= & 388,511 \frac{1}{T}-7,169\left(\log T+118,628 \frac{1}{T}\right) \\
& +20,437
\end{aligned}
$$

darstellen (Abb. 3). Zum Vergleich ist in Abb. 3 der

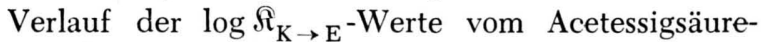
äthylester ${ }^{1}$ mit eingezeichnet.

Aus Gl. (1) ergibt sich die Enthalpieänderung der Keto-Enol-Umwandlung zu

${ }^{5}$ B. Eistert, F. Arndt, L. Löwe u. E.Ayca, Chem. Ber. 84, 156 [1951].

${ }^{6} \vartheta=273,20^{\circ} \mathrm{K}$.

$$
\begin{aligned}
\Delta H_{T_{\mathrm{K} / \mathrm{E}}}=\Delta H_{\vartheta}+\Delta C_{p}(T-\vartheta) & \\
& =-1777-14,238(T-\vartheta) .
\end{aligned}
$$

In Tab. 2 sind die berechneten thermodynamischen Daten $\Delta G, \Delta H, T \Delta S$ und $\Delta S$ für Temperaturintervalle von je $20^{\circ}$ angegeben.

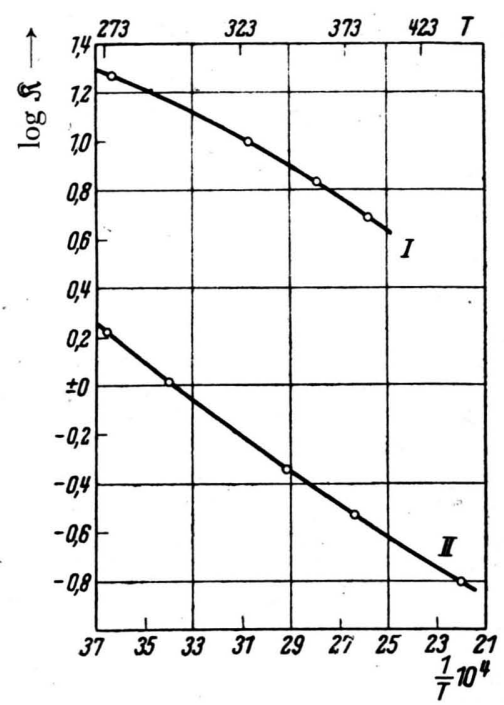

Abb. 3. $\log \Omega_{\mathrm{K} \rightarrow \mathrm{E}}$ als Funktion von $1 / T$. $\circ$ Meßpunkte. I: Acetylaceton. II: Acetessigester. Die ausgezogenen Kurven sind berechnet.

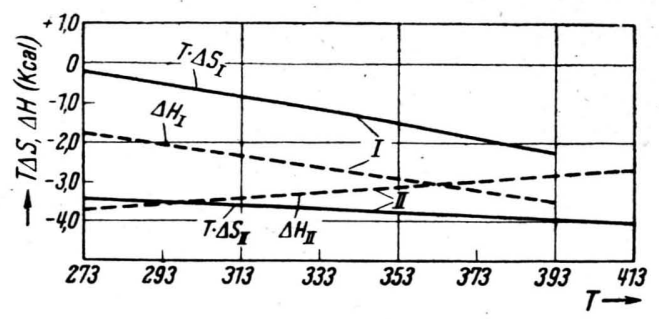

Abb. 4. $\Delta H_{T_{\mathrm{K} \rightarrow \mathrm{E}}}$ und $\mathrm{T} \Delta \mathrm{S}$ als Funktion der Temperatur für Acetylaceton (I) und Acetessigsäureäthylester (II). Ausgezogene Kurven $T \Delta S$, gestrichelte Kurven $\Delta H$.

Der Verlauf der Enthalpie- und Entropieänderung mit der Temperatur ist in Abb. 4 für das Acetylaceton und zum Vergleich für den Acetessigsäureäthylester dargestellt.

Die freie Bildungsenthalpie $\Delta G$ wurde aus der K-E-Gleichgewichtskonstanten nach

$$
\Delta G=-R T \ln \Re
$$

berechnet. Mit den erhaltenen Werten konnte dann die Entropieänderung $\Delta S$ nach Gl. (3) erhalten werden.

$$
T \Delta S=\Delta H-\Delta G .
$$




\begin{tabular}{|c|c|c|c|c|c|c|}
\hline$T$ & $\%_{0} E$ & $\log K_{\mathrm{K} \rightarrow \mathrm{E}}$ & $\begin{array}{c}\Delta H \\
\mathrm{kcal}\end{array}$ & $\begin{array}{c}\Delta G \\
\mathrm{kcal}\end{array}$ & $\begin{array}{c}T \Delta S \\
\mathrm{kcal}\end{array}$ & $\begin{array}{c}\Delta S \\
\mathrm{cal} / \mathrm{Grad}\end{array}$ \\
\hline$(1)$ & $(2)$ & $(3)$ & $(4)$ & $(5)$ & $(6)$ & $(7)$ \\
273,2 & 95,0 & 1,2780 & $-1,777$ & $-1,597$ & $-0,180$ & $-0,66$ \\
293,2 & 93,7 & 1,1734 & $-2,062$ & $-1,574$ & $-0,488$ & $-1,66$ \\
313,2 & 92,1 & 1,0685 & $-2,847$ & $-1,531$ & $-0,816$ & $-2,61$ \\
333,2 & 90,2 & 0,9643 & $-2,631$ & $-1,469$ & $-1,162$ & $-3,49$ \\
353,2 & 87,9 & 0,8613 & $-2,916$ & $-1,392$ & $-1,524$ & $-4,31$ \\
373,2 & 85,2 & 0,7599 & $-3,201$ & $-1,297$ & $-1,904$ & $-5,10$ \\
393,2 & 82,0 & 0,6604 & $-3,486$ & $-1,188$ & $-2,298$ & $-5,84$ \\
413,2 & 78,45 & 0,5627 & $-3,770$ & $-1,063$ & $-2,707$ & $-6,55$ \\
\hline
\end{tabular}

Tab. 2. Thermodynamische Daten für die $\mathrm{K} \rightarrow$ E-Umwandlung von Acetylaceton im Gaszustand für $T$-Intervalle von je $20^{\circ}$.

Aus Tab. 1 und Abb. 3 ist folgendes zu ersehen:

1. Acetylaceton hat im gesamten $T$-Bereich einen höheren Enolgehalt als Acetessigester.

2. Der Enolgehalt nimmt bei Acetylaceton mit steigender Temperatur weniger ab als bei Acetessigester.

3. $\mathrm{Da}$ bei Acetylaceton die Enolisierungstendenz größer ist als bei Acetessigester, liegt an folgendem:

a) $|T \Delta S|$ ist bei Acetylaceton stets $<|\Delta H|$, und

b) die ( $T \Delta S ; T$-) und ( $\Delta H, T$-) Geraden divergieren nur wenig. Bei Acetessigester dagegen überschneiden sich die $(T \Delta S, T$-) und $(\Delta H, T$-) Geraden. Für alle Temperaturen $T>293^{\circ}$ ist, im Gegensatz zu Acetylaceton $|T \Delta S|>|\Delta H|$, so daß die Entropieabnahme bei der $\mathrm{K} \rightarrow \mathrm{E}$-Umwandlung sich bei Acetessigester ungünstiger auf $\Delta G$ auswirkt als bei Acetylaceton ${ }^{7}$.

Wir danken der Deut schen Forschungs$\mathrm{g}$ e meinschaft für die Úberlassung von Apparaten und dem Würzburger Universitätsbund für die Bewilligung von Mitteln zur Durchführung der Untersuchungen.

7 Eine ausführliche, prinzipielle Betrachtung zur Theorie und Thermodynamik der K-E-Umwandlung auch bei substituierten Acetessigestern erfolgt in einer besonderen Arbeit: G. Briegle b u. W. S to ckmei er, Z. angew. Chem. 64 [1952].

\title{
Ein neues Verfahren bei der Trennung von gelösten Stoffen durch Thermodiffusion in der Flüssigkeit
}

\author{
Von H. Korsching \\ Aus dem Max-Planck-Institut für Physik, Göttingen \\ (Z. Naturforschg. 7 b, 187-191 [1952]; eingegangen am 10. Dezember 1951)
}

Hern Professor Werner Heisen berg zum 50. Geburtstag

Es wird eine Methode angegeben, die die Trennung zweier gelöster Stoffe in gleiche Anteile mit etwa gleicher Trennung erlaubt. Eine dazu geeignete Apparatur wird angegeben und ihre Wirkungsweise bei dem Gemisch Phenanthren-9-Brom-phenanthren in Benzol beschrieben.

$\mathrm{W}$ ird man vor die Aufgabe gestellt, zwei Stoffe zu trennen, so wird in vielen Fällen die Thermodiffusion, kombiniert mit Siphonströmung, geeignet sein. Ist das Stoffgemisch flüssig, so läßt es sich leicht auf diese Weise in zwei Anteile zerlegen, bei denen eine teilweise oder auch vollständige Trennung erfolgt ist. Mitunter genügen kleine Unterschiede des
Volumens der zu trennenden Moleküle, um zu einem praktisch verwertbaren Ergebnis zu kommen. So trennt sich beispielsweise das Isomerenpaar Dioxan -Glykolacetal so gut, daß mehrmalige Wiederholung des Prozesses eine vollständige Trennung erreichen ließe. Ist aber das zu trennende Stoffgemisch fest und hat einen hohen Schmelzpunkt, oder zersetzt es sich 